\title{
Gene transfer by viral vectors into blood vessels in a rat model of retinopathy of prematurity
}

Itay Chowers, Eyal Banin, Yitzchak Hemo, Rinat Porat, Haya Falk, Eli Keshet, Jacob Pe'er, Amos Panet

\begin{abstract}
Aims-To test the feasibility of gene transfer into hyaloid blood vessels and into preretinal neovascularisation in a rat model of retinopathy of prematurity (ROP), using different viral vectors.

Methods-Newborn rats were exposed to alternating hypoxic and hyperoxic conditions in order to induce ocular neovascularisation (ROP rats). Adenovirus, herpes simplex, vaccinia, and retroviral (MuLV based) vectors, all carrying the $\beta$ galactosidase $(\beta$-gal) gene, were injected intravitreally on postnatal day 18 (P18). Two sets of controls were also examined: P18 ROP rats injected with saline and $P 18$ rats that were raised in room air before the viral vectors or saline were injected. Two days after injection, the rats were killed, eyes enucleated, and $\beta$-gal expression was examined by $\mathrm{X}$-gal staining in whole mounts and in histological sections.

Results-Intravitreal injection of the adenovirus and vaccinia vectors yielded marked $\beta$-gal expression in hyaloid blood vessels in the rat ROP model. Retinal expression of $\beta$-gal with these vectors was limited almost exclusively to the vicinity of the injection site. Injection of herpes simplex yielded a punctuate pattern of $\beta$-gal expression in the retina but not in blood vessels. No significant $\beta$-gal expression occurred in rat eyes injected with the retroviral vector.

Conclusions-Adenovirus is an efficient vector for gene transfer into blood vessels in an animal model of ROP. This may be a first step towards utilising gene transfer as a tool for modulating ocular neovascularisation for experimental and therapeutic purposes.

(Br f Ophthalmol 2001;85:991-995)
\end{abstract}

In animal models, modulation of retinal neovascularisation can be achieved by administration of various agonists or antagonists to angiogenesis such as vascular endothelial growth factor (VEGF), growth hormone, and others. ${ }^{1-6}$ Additional novel investigative methods applied in the study of ocular angiogenesis are the use of transgenic animals and gene knockout techniques. For example, vascular endothelial growth factor (VEGF) receptors, and fibroblast growth factor (FGF) knockout mice, as well as transgenic mice that overexpress VEGF or FGF have been extensively studied. ${ }^{7-9}$ These animal models have greatly increased our knowledge of the importance of factors such as VEGF or FGF in retinal neovascularisation. However, the establishment of such lines of mice is quite difficult, and this approach cannot be directly used as a therapeutic modality in humans.

An alternative method to study the effects of a gene product is to deliver and express the gene in the target tissue by a viral vector. This approach has been used extensively in the study of ocular diseases, especially retinal degenerations, in animal models. ${ }^{10-16}$ To the best of our knowledge, gene delivery to ocular blood vessels has not been previously described.

The purpose of the present study was to examine the feasibility of gene transfer by four different viral vectors into hyaloid and preretinal blood vessels in the rat ROP model. Furthermore, ocular tissue tropism of these viral vectors was investigated.

\section{Methods}

RAT ROP MODEL

Animals were treated according to the ARVO statement for the use of animals in ophthalmic and vision research. Abnormal ocular neovascularisation was induced in newborn rats as described by Penn et al ("rat ROP model"). ${ }^{17}$ Postnatal day 1 (P1) Sabra rats and their mothers were raised for 14 days in alternating, 24 hour cycle, hypoxic (10\% oxygen) and hyperoxic ( $70 \%$ oxygen) environments. At P14 rats were removed from the incubator to room air. Intravitreal injections were performed 4 days later (P18). P18 Sabra rats that were raised in room air served as controls.

\section{VIRAL VECTORS}

Four different viral vectors were used:

(1) Adenoviral vector containing the $\beta$ galactosidase $(\beta$-gal) reporter gene under the control of the cytomegalovirus (CMV) promoter (Ad5CMVlacZ). This adeno vector is E1A defective. The replication defective virus was propagated in 293 cells. ${ }^{18}$ 
(2) The herpes simplex virus type I (HSV) vector contained the reporter gene $\beta$-gal (tkLTRZ1) under control of the murine leukaemia virus (MuLV) LTR promoter inserted at the thymidine kinase (tk) gene locus. ${ }^{19}$ The virus is replication competent in dividing cells and it was grown on CV1 monkey cells.

(3) The vaccinia expression vector, encoding the $\beta$-gal gene under control of the vaccinia early promoter (vSC9). ${ }^{20}$ The virus is replication competent and was propagated in $\mathrm{HeLa}$ cells.

All the DNA viruses were titrated (cell forming units, CFU) in parallel on HeLa cells using the $\mathrm{X}$-gal staining technique.

(4) The $\beta$-gal encoding recombinant retrovirus (pCLMGF-LacZ) was constructed using the pLXSN vector with the $\beta$-gal gene under control of the MuLV LTR promoter. The transfer vector was packaged by co-transfection with the pCL-Eco packaging construct in 293 cells. $^{21}$

Virus supernatants were collected after 2 days and the virus titre (CFU) was determined using NIH-3T3 cells and X-gal staining.
INTRAVITREAL INJECTIONS

At P18 rats were anaesthetised in an ether chamber. Borosilicate glass pipettes $(1.2 \mathrm{~mm}$ external diameter, $0.69 \mathrm{~mm}$ internal diameter, Sutter Instruments Co, Navato, CA, USA) were pulled to form $50 \mu \mathrm{m}$ calibre tips by an electrode puller (P-97, Sutter Instruments Co, CA, USA) and were connected to a microinjection unit (PLI-100, Medical Systems Corp, Greenvale, NY, USA) for intravitreal injections. All injections were performed under magnification, using a binocular microscope and a micromanipulator.

A volume of $1 \mu \mathrm{l}$ containing the same cell forming units $\left(10^{5} \mathrm{CFU}\right)$ of one of the viral vectors (adenovirus, herpes simplex, or vaccinia) or $10^{4} \mathrm{CFU}$ of the retroviral vector were injected intravitreally to $10 \mathrm{ROP}$ rats via the pars plana in one eye. Two control groups were studied. The first included 10 P18 ROP rats in whom saline was injected instead of the viral vectors. The second control group included 25 P18 rats that were raised in room air. The four viral vectors and saline were each injected intravitreally in five such rats.
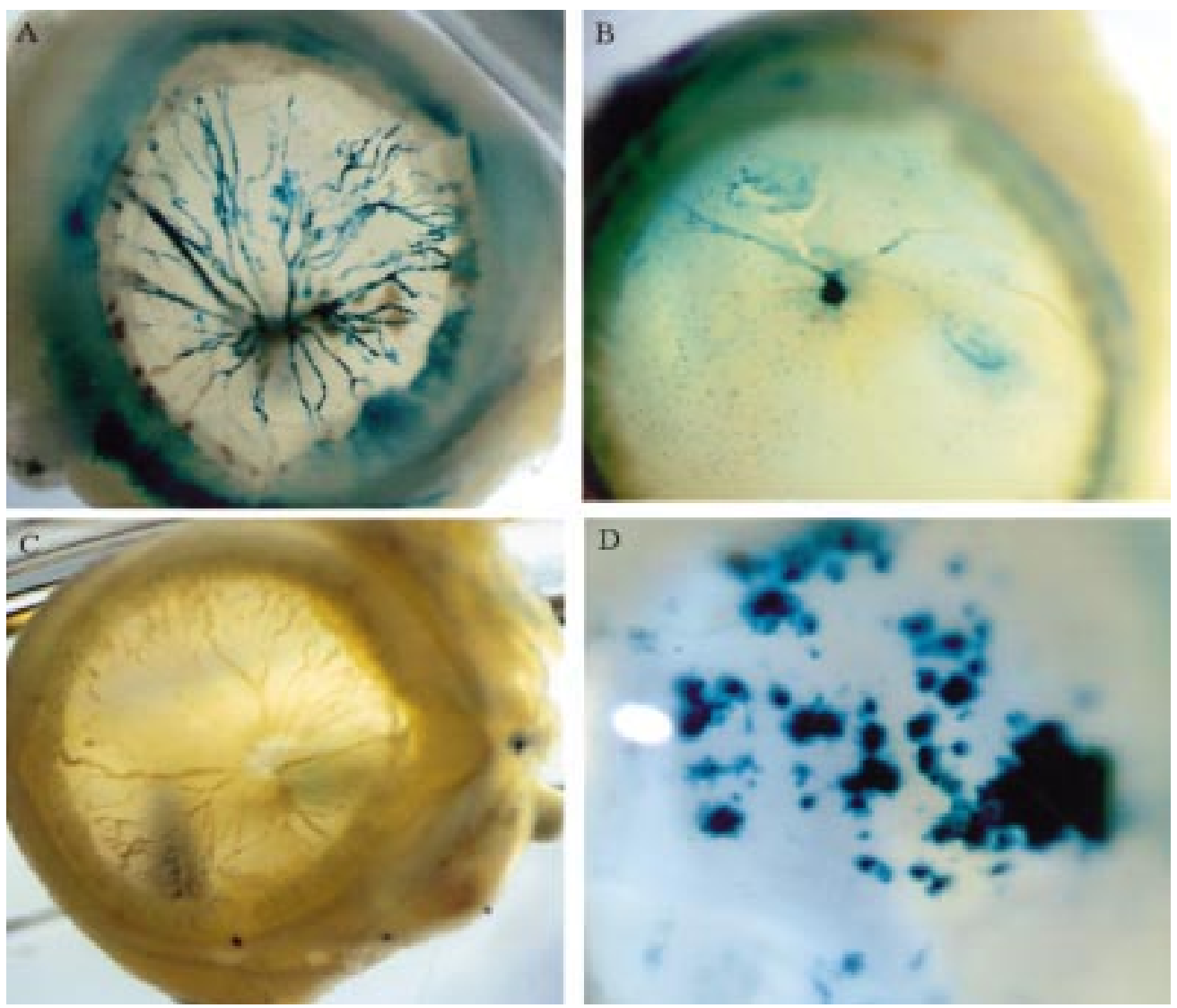

Figure 1 Macroscopic photographs of eyes enucleated from normal and ROP P20 rats after intravitreal injection of $\beta$-gal carrying viral vectors. ROP rats underwent intravitreal injection at P18 with adenovirus vector (10 infectious units) (A) (original magnification $\times 3$ ), saline $(C)$ (original magnification $\times 3$ ), or herpes vector $\left(10^{5}\right.$ infectious units) (D) (original magnification $\times 5$ ). Normal $P 18$ rats were injected with adenovirus vector (105 infectious units) (B) (original magnification $\times 4)$. ROP and normal rats were also injected with vaccinia and retroviral vector (not shown). After enucleation, the cornea, iris, and lens were removed and the eye cup was fixed in $0.2 \%$ glutaraldehyde, $2 \%$ formaldehyde, 2 $m M \mathrm{MgCl}_{2}, 0.05 \mathrm{M} \mathrm{NaPO}_{4}$ buffer ( $p H$ 7.4), followed by overnight X-gal staining. Adenovirus injected eye $(A)$ shows extensive $\beta$-gal expression (blue stain) in the hyaloid system; notice the paucity of retinal staining. Normal P2O rat eye injected with adenovirus vector shows $X$-gal staining of the remnants of the hyaloid system as well as areas of retinal staining (B). Herpes injected eye shows punctate staining of the retina (D). Control, saline injected ROP rat eye shows no staining of the hyaloid or retina $(C)$. 
X-GAL STAINING

At P20, rats were killed, eyes were enucleated, and the cornea and lens removed. The remaining eye cups were fixed in $0.2 \%$ glutaraldehyde, $2 \%$ formaldehyde, $2 \mathrm{mM} \mathrm{MgCl}{ }_{2}, 0.05 \mathrm{M}$ $\mathrm{NaPO}_{4}$ buffer ( $\mathrm{pH} 7.4$ ), washed three times in $2 \mathrm{mM} \mathrm{MgCl}_{2}, 0.02 \% \mathrm{NP} 40,0.05 \mathrm{M} \mathrm{NaPO}$ buffer, and incubated overnight at $37^{\circ} \mathrm{C}$ with 5 $\mathrm{mM}$ potassium ferrocyanide, $2 \mathrm{mM} \mathrm{MgCl}_{2}$, and $20 \mathrm{mg} / \mathrm{ml} \mathrm{X}$-gal (5-bromo-4-chloro-3indolyl $\beta$-D-galactopyranoside) in $0.05 \mathrm{M}$ $\mathrm{NaPO}_{4}$ buffer. Eye cups were then either fixed in formalin, embedded in paraffin, cut into 4 $\mu \mathrm{m}$ sections and stained with haematoxylin and eosin or, alternatively, the retina was separated and a whole mount preparation was examined under a microscope.

\section{Results}

Rats that were maintained until postnatal day 14 in alternating hypoxic and hyperoxic conditions manifested at P20 the ocular blood vessel abnormalities previously described in this animal model of ROP. ${ }^{17}$ Marked congestion and tortuosity of the retinal and iris vessels were observed along with preretinal haemorrhages and persistence of the hyaloid system. Preretinal vessels ("neovascular tufts") emerging from the retinal circulation were detected in histological sections in two eyes. In rats maintained in room air, the normal postnatal process of hyaloid system regression and retinal vascular development was observed. At P20, only remnants of the hyaloid system could be seen.

The most extensive expression of $\beta$-gal in blood vessels occurred following intravitreal injection of the adenovirus vector

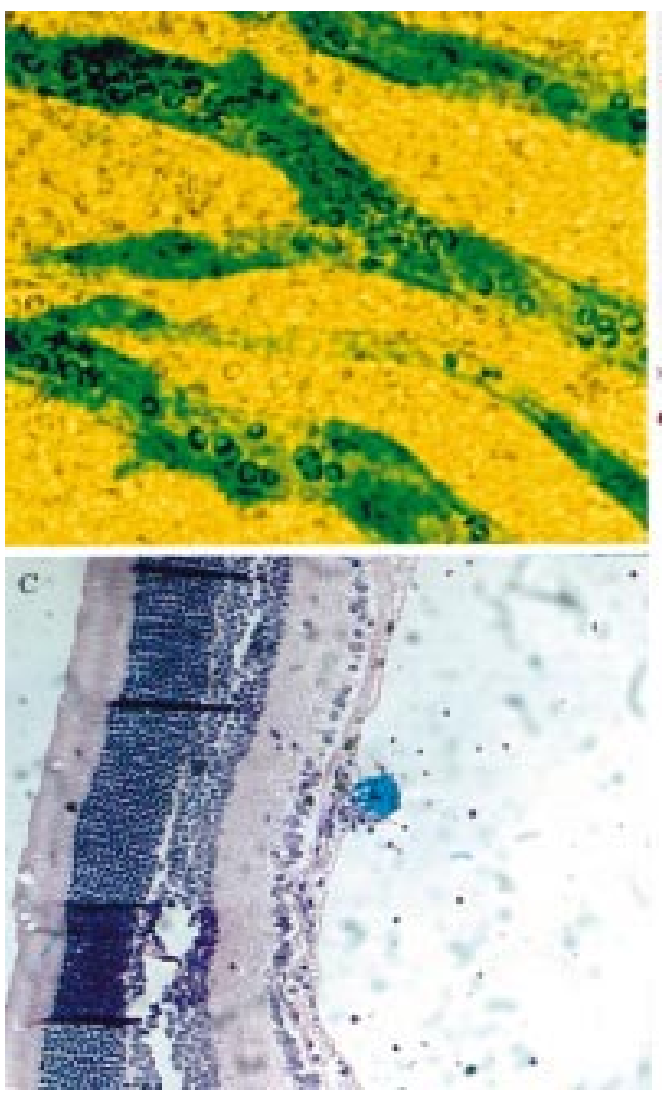

(Ad5CMVlacZ) to ROP rats. There was marked X-gal staining of the hyaloid system in all 10 animals of this group (Fig 1A). Macroscopically, the whole hyaloid system seemed to express $\beta$-gal, resembling large blue "tree trunks" inserting into the optic disc (Fig 1A). Microscopically, histological sections and whole mount preparations showed $\beta$-gal expression in the walls of hyaloid blood vessels (Fig 2A and B). By contrast, retinal blood vessels did not show significant levels of $\beta$-gal expression. Histological sections revealed preretinal "neovascular tufts" emerging from retinal blood vessels in two of the 10 ROP rats that were injected with adenovirus. In both cases, $\beta$-gal expression was seen in these preretinal tufts. Interestingly, the staining seems to stop abruptly at the point at which the vessels originate from the retina. The blood vessels within the retina in this area showed no $\beta$-gal expression (Fig 2C). In general, retinal expression of $\beta$-gal (as opposed to the hyaloid system) was almost exclusively limited to the area of injection (at the pars plana). Here, neuroretinal elements other than blood vessels, such as the inner nuclear layer and ganglion cells, stained as well. Histologically, retinal structure and cellular components were well preserved in all injected animals.

Vaccinia (vSC9) injection yielded $\beta$-gal expression only in segments of the hyaloid system (as opposed to staining of the whole hyaloid system in the adenovirus injected ROP rats) in three of the 10 ROP rats but not in the five normal P18 rats; retinal staining was again mainly limited to the injection site. The HSV vector (tkLTRZ1) expressed $\beta$-gal almost

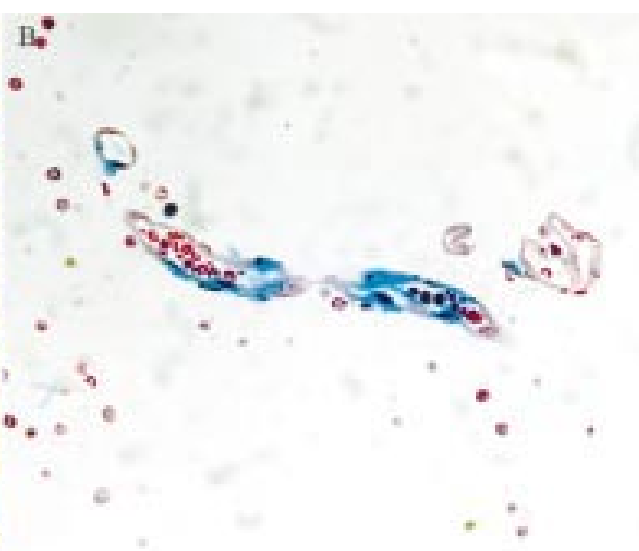

Figure 2 Histological sections and whole mount preparation of adenovirus injected eye from P20 ROP rat. Retinal whole mount at high magnification $(\times 40)$ shows $\beta$-gal expression in hyaloid vessels $(A)$; note red blood cells in the vessel lumen demonstrating patency of the hyaloid at this stage. Formalin fixed, paraffin embedded sections stained with haematoxylin and eosin show $\beta$-gal expression in hyaloid vessel walls (B) and in a preretinal neovascular tuft $(C)$; note that within the retina, the vessel from which the tuft originates and the surrounding cells did not stain $(C)$. 
exclusively in the retina, in the form of punctate staining (Fig 1D), and histological sections revealed $\beta$-gal expressing cells in the inner retina. The hyaloid system did not stain with X-gal in any of the 10 ROP rats or in the five normal P18 rats infected with the HSV vector. Intravitreal injection of the retroviral vector (pCLMGF-LacZ) did not yield any retinal or hyaloid expression of $\beta$-gal.

In the eyes of normal P18 rats injected with adenovirus, $\beta$-gal expression was observed in the remnants of the regressing hyaloid system as well as at the injection site in all five rats. Interestingly, in these normal rats, gene delivery into the retina was more extensive as compared with the ROP rats (Fig 1B).

Additional ocular structures that showed $\beta$-gal expression after injection of adenovirus, vaccinia, or herpes viral vectors were the ciliary body, pigment epithelium of the iris, and the corneal endothelium. These three vectors, but not the retrovirus, caused a severe vitreal and anterior chamber inflammatory reaction that was first noted approximately 48 hours following injection. Eyes of ROP rats and normal P18 rats that were injected with saline showed no $\mathrm{X}$-gal staining or inflammation (Fig 1C).

\section{Discussion}

In this study, the feasibility of gene delivery to hyaloid and preretinal blood vessels in a rat model of ROP was tested. Our results show that adenovirus vector can efficiently deliver genes into hyaloid blood vessels in the rat ROP model. Although the constitutive CMV promoter was used to control gene expression in this vector, a surprising degree of specificity of $\beta$-gal expression was observed in hyaloid blood vessels. In the retina, adeno mediated $\beta$-gal expression was limited almost exclusively to the injection site.

Several factors may contribute to the relatively limited adenovirus vector single cycle infection of the retina. It is possible that the inner limiting membrane and the posterior vitreous face act as a barrier, physically blocking infection of the retina itself. Indeed, other investigators have shown that when retinal expression is the goal, subretinal injection of the viral vectors is preferable to intravitreal injection. ${ }^{22}$ In human vascular retinopathies, splitting of the posterior cortical vitreous, or posterior vitreoschisis, is a common finding that is usually manifested as two dense vitreous membranes. ${ }^{23}$ We speculate that a similar condition may exist in the rat ROP model, adding another obstacle (apart from the inner limiting membrane of the retina) between the vitreous cavity and the retina. Our observation of more extensive retinal expression of the trans-gene in the normal rat retina compared with the ROP rat retina seems to support the possibility of such preferential accessibility.

Another factor influencing retinal trans-gene expression is viral vector multiplicity of infection. Higher concentrations of the vector would perhaps deliver genes more efficiently to the retina, and it is possible that the multiplicity of infection used (which we tried to keep low in order to decrease levels of inflammation) contributed to the apparent specificity of expression in the hyaloid.

Infection by a viral vector such as adenovirus may damage the cell even without expressing the trans-gene. Therefore, the relatively tissue specific gene delivery demonstrated in our study is encouraging, since when gene delivery into hyaloid or preretinal blood vessels is the goal, prevention of infection of the retina is desirable.

The other viral vectors tested in this study showed either partial (vaccinia) or no (retrovirus and HSV) gene transfer to the hyaloid vessels. The differing pattern of reporter gene expression by the various vectors is probably due to differences in their ability to infect specific tissues, since the promoters driving the reporter gene are constitutive and do not confer tissue specificity. It should be noted that the four viral vectors tested have different replication cycles and different promoters to drive trans-gene transcription. Nevertheless, when $\beta$-gal enzyme activity was measured by the ONPG colorimetric test in extracts of a cell line, mouse NIH 3T3, infected at the same viral multiplicity, expression levels were very similar for the three DNA viruses and only fivefold lower per cell for the retroviral vector (unpublished data). Therefore, vector tissue tropism appears to be related to early steps of infection which may explain the differences in pattern of expression between the vectors. For instance, HSV receptors may be missing in vitreal blood vessels, preventing infection of the endothelium by this vector. ${ }^{25}{ }^{26}$ The retroviral vector and to some extent the HSV vector require cell proliferation in the target tissue in order to achieve infection. The lack of endothelial cell proliferation in the hyaloid vessels at P18 might be the critical factor contributing to the failure of gene delivery by these two vectors. Thus, the results presented in this report most probably reflect tissue tropism of the four viral vectors in the eye, and are probably not due to differences in trans-gene promoter activity among the viruses.

As opposed to the persistence of the hyaloid system that was markedly enhanced in our ROP rats, only two preretinal neovascular tufts were observed. Therefore, although both expressed $\beta$-gal, conclusions regarding efficacy of gene delivery to such preretinal tufts cannot be drawn directly. However, the hyaloid system, being a vascular system in the vitreous cavity, simulates some aspects of neovascularisation on the disc and pre-retinal neovascularisation. The hyaloid is also intriguing from a developmental aspect, affording a model in which not only the process of blood vessel growth but also that of normal vessel regression can be studied. The hyaloid system, which in humans regresses before birth, is normally present in the first few weeks of life in the rat. ${ }^{27}$ This system is composed of non-fenestrated capillaries as well as larger blood vessels, all surrounded by pericytes ${ }^{28}$ VEGF serves as a survival factor for the hyaloid system, ${ }^{29}$ and in the rat, hyaloid capillaries continue to show sprouting after birth. ${ }^{30}$ This depends on the postnatal age of 
the animal and the oxygen levels in which the animal is kept. At the other end of the process, apoptosis has a role in the normal regression of the hyaloid system. ${ }^{29}$ Therefore, delivery of genes into the hyaloid system may serve to study both angiogenesis and apoptosis of blood vessels.

Specific expression of genes in vascular endothelial cells is possible by using vectors in which the genes are under the regulation of an endothelium specific promoter. ${ }^{31-34}$ In the eye, genes such as the $67 \mathrm{kD}$ laminin receptor, that are expressed preferentially in proliferating and not in quiescent retinal blood vessels, have been identified, and their nucleotid sequence has been determined. ${ }^{3}$ In the future, gene delivery and specific expression in the endothelium of proliferating ocular vessels can be attempted by keeping the delivered gene under the regulation of an endothelium specific promoter. Such a method can be used experimentally to assess the effect of different genes on blood vessels.

In addition, by using the strategy of suicide gene delivery, ocular neovascular tissue could be preferentially targeted and destroyed. For example, new preretinal (or choroidal) blood vessels may be infected with a vector carrying the herpes simplex thymidine kinase under the control of a promoter that activates genes only in proliferating endothelium. Gene delivery will be followed by treatment with ganciclovir, thereby activating and specifically affecting proliferating blood vessels. Such suicide gene delivery in the eye (into tissues other than blood vessels) has been used to inhibit RPE cell growth in vitro, ${ }^{35}$ and to treat experimental proliferative vitreoretinopathy in the rabbit. ${ }^{136}$

In conclusion, our study demonstrates the feasibility of gene transfer by viral vectors into hyaloid blood vessels in an animal model of ischaemia induced vitreoretinopathy. Relative specificity of expression was observed, perhaps because of preferential accessibility of some of the viral vectors into these hyaloid blood vessels. Based on these results, and considering the available techniques in gene targeting, the concept of modulating ocular neovascularisation by gene delivery should be tested.

Supported in part by the Yedidut, Yael, and Hebrew University - Hadassah research grants.

1 Robinson GS, Pierce EA, Rook SL, et al. Oligodeoxynucleotides inhibit retinal neovascularization in a murine mode of proliferative retinopathy. Proc Natl Acad Sci 1996;93:4851-6

2 Smith LE, Kopchick JJ, Chen W, et al. Essential role of growth hormone in ischemia-induced retinal neovascularization. Science 1997;276:1706-9.

3 Stitt AW, McKenna D, Simpson DA, et al. The 67-kd laminin receptor is preferentially expressed by proliferating retinal vessels in a murine model of ischemic retinopathy. Am f Pathol 1998;152:1359-65.

4 Yoshida A, Yoshida S, Khalil AK, et al. Role of NF-kappaBmediated interleukin-8 expression in intraocular neovascu-

5 Alon T, Hemo I, Itin A, et al. Vascular endothelial growth factor acts as a survival factor for newly formed retinal vessels and has implications for retinopathy of prematurity. Nat Med 1995;1:1024-8.

6 Seo M, Kwak N, Ozaki H, et al. Dramatic inhibition of retiSeo $\mathrm{M}$, Kwak N, Ozaki $\mathrm{H}$, et al. Dramatic inhibition of reti-
nal and choroidal neovascularization by oral administration nal and choroidal neovascularization by oral administra
of a kinase inhibitor. Am $\mathcal{F}$ Pathol 1999;154:1743-53.

7 Shalaby F, Rossant J, Yamaguchi T, et al. Failure of blood island formation and vasculogenesis in Flk-1-deficien mice. Nature 1995;376:62-6.
8 Ozaki $\mathrm{H}$, Okamoto $\mathrm{N}$, Ortega S, et al. Basic fibroblast growth factor is neither necessary nor sufficient for the development of retinal neovascularization. Am f Pathol 1998;153:757-65.

9 Okomoto N, Trobe T, Hackett S, et al. Transgenic mice with increased expression of VEGF in the retina: a new model of retinal neovascularization. Am F Pathol 1997;151:281-91.

10 Bennett J, Pakola S, Zeng Y, et al. Humoral response after administration of E1-deleted adenoviruses: immune privi9.

11 Bennett J, Tanabe T, Sun D, et al. Photoreceptor cell rescue in retinal degeneration (rd) mice by in vivo gene therapy. Nat Med 1996;2:649-54.

12 Hoffman L, Maguire A, Bennett J. Cell-mediated immune response and stability of intraocular transgene expression response and stability of intraocular transgene expression
after adenovirus-mediated delivery. Invest Ophthalmol Vis after adenovirus-mediat
Sci 1997;38:2224-33.

13 Kimura H, Sakamoto T, Cardillo JA, et al. Retrovirusmediated suicide gene transduction in the vitreous. Hum Gene Ther 1996;7:799-808.

14 Larkin DF, Oral HB, Ring CJ, et al. Adenovirus-mediated gene delivery to the corneal endothelium. Transplantation 1996;61:363-70.

15 Mashhour B, Couton D, Perricaudet $M$, et al. In-vivo adenovirus-mediated gene transfer into ocular tissues. Gene Ther 1994;1:122-6.

16 Murata T, Kimura H, Sakamoto T, et al. Ocular gene therapy: experimental studies and clinical possibilities. Ophthalmic Res 1997;29:242-51.

17 Penn J, Henry M, Tolman B. Exposure to alternating hypoxia and hyperoxia causes severe proliferative retinopathy in the newborn rat. Pediater Res 1994;36:724-31.

18 Graham F, Prevec L. Manipulation of adenovirus vectors. Gene transfer and expression protocols. In: Murray E, Walker J, eds. Methods in molecular biology. Clifton, NJ: Humana Press, 1991:vol 7.

19 Davar G, Kramer M, Garber D, et al. Comparative efficacy of expression of genes delivered to mouse sensory neurons with herpes virus vectors. F Comp Neuro 1994;339:3-11.

20 Chakrabarti S, Brechling K, Moss B. Vaccinia virus expression vector: coexpression of beta-galactosidase provides 1985;5:3403-9.

21 Naviaux R, Costanzi E, Haas M, et al. The pCL vector system: rapid production of helper-free, high-titer, recombinant retroviruses. 7 Virol 1996;70:5701-5.

22 Ali R, Reichel M, Alwis MD, et al. Adeno-associated virus gene transfer to mouse retina. Hum Gene Ther 1998;9:81gen

23 Kakehashi A, Schepens CL, de Sousa Neto A, et al. Biomicroscopic findings of posterior vitreoschisis. Ophthalmic Surg 1993;24:846-50.

24 Schwatz SD, Alexander R, Hiscott P, et al. Recognition of vitreoschisis in proliferative diabetic retinopathy. A useful landmark in vitrectomy for diabetic traction retinal detachment. Ophthalmology 1996;103:323-8.

25 Shukla D, Liu J, Blaiklock P, et al. A novel role for 3-O-sulfated heparan sulfate in herpes simplex virus entry. Cell 1999;99:13-22.

26 Cocchi F, Menotti L, Dubreuil P, et al. Cell-to-cell spread of wild-type herpes simplex virus type 1 , but not of syncytial strains, is mediated by the immunoglobulin-like receptors that mediate virion entry, nectin 1 (PRR1/HveC/HIgR) and nectin2 (PRR2/HveB). F Virol 2000;74:3909-17.

27 Latker C, Kuwabara T. Regression of the tunica vasculosa lentis in the postnatal rat. Invest Ophthalmol Vis Sci 1981;21:689-99.

28 Zhu M, Provis JM, Penfold PL. The human hyaloid system: cellular phenotypes and inter-relationships. Exp Eye Res 1999;68:553-63.

29 Mitchell C, Risau W, Drexler H. Regression of vessels in the tunica vasculosa lentis is initiated by coordinated endothelial apoptosis: a role for vascular endothelial growth factor as a survival factor for endothelium. Dev Dyn 1998;213: 322-33.

30 Wang J, Toida K, Uehara Y. The tunica vasculosa lentis; an expedient system for studying vascular formation and regression. F Electron Microsc (Tokyo) 1990;39:46-9.

31 Ozaki K, Yoshida T, Ide H, et al. Use of von Willebrand factor promoter to transduce suicidal gene to human tor promoter to transduce suicidal gene to human
endothelial cells, HUVEC. Hum Gene Ther 1996;7:148390 .

32 Wickham TJ, Haskard D, Segal D, et al. Targeting endothelium for gene therapy via receptors up-regulated during angiogenesis and inflammation. Cancer Immunol Iтmиnother 1997;45:149-51

33 Walton T, Wang JL, Ribas A, et al. Endothelium-specific expression of an E-selectin promoter. Anticancer Res 1998; 18:1357-60

34 Jaggar RT, Chan HY, Harris AL, et al. Endothelial cell-specific expression of tumor necrosis factor-alpha from the KDR or E-selectin promoters following retroviral delivery. Hum Gene Ther 1997;8:2239-47.

35 Schubert CA, Kimura H, Spee C, et al. Retrovirus-mediated transfer of the suicide gene into retinal epithelial cells in transfer of the suicide gene into retin

36 Sakamoto T, Kimura H, Scuric Z, et al. Inhibition of experimental proliferative vitreoretinopathy by retroviral vectormediated transfer of suicide gene. Can proliferative vitreoretinopathy be a target of gene therapy? Ophthalmology 1995;102:1417-24. 